\title{
On The Solutions of Some Difference Equations Systems and Analytical Properties
}

\author{
Münevver Tuz ${ }^{1}$ \\ ${ }^{1}$ Department of Mathematics, University of Firat, Elazığ, Turkey \\ maydin23@yahoo.com
}

\begin{abstract}
Absract. In this study, we investigated the global asymptotic behaviors of their solutions by taking the second-order difference equation system. According to the given conditions, we obtained some asymptotic results for the positive balance of the system. We have also worked on q-fast changing functions. Such functions form the class of $q$-Caramate functions. We have applied $q$-Caramate functions to linear $q$-difference equations and We have also learned about the asymptotic behavior of solutions. In addition, we have studied the problem of initial and boundary value for the $q$-difference equation
\end{abstract}

\section{Introduction}

Difference equations (ordinary differential and $q$-differential) since ancient times are equations of interest to mathematicians and physicists. These equations solve the differential equations by shaping them into mathematical models of many other practical phenomena. Difference equations can be easily algorithmized and solved on a computer they are very suitable for.

Studies on q-difference equations appeared already at the beginning of the last century in intensive works especially by Adams[1], Atkinson[2], Bairamov[3,4], Jackson[5], Elaydi[6], Guseinov[7,8], Carmichael, Trjitzinsky and others. Unfortunately, from the thirties up to the beginning of the eighties, only nonsignificant interest in the area was observed. Since years eighties, an intensive and somewhat surprising interest in the subject reappeared in many areas of mathematics and applications including mainly new difference calculus and orthogonal polynomials, $q$-combinatorics, $q$-arithmetics, $q$-integrable systems and variational $q$ calculus.

\section{Preliminaries}

We have investigated the global asymptotic behavior of the solutions of the system of the difference equation

$$
\begin{gathered}
k_{n} \frac{d^{2} y_{n}(t)}{d t^{2}}=h_{n} \frac{d y_{n}(t)}{d t}+a_{n-1} y_{n-1}(t)+b_{n} y_{n}(t)+q a_{n} y_{n+1}(t) \\
n \in\{0,1 \ldots N-1\}, t \geq 0,
\end{gathered}
$$

where $\mathrm{N} \geq 2$ is a fixed integer and $q>1$ is a fixed real number.

$$
\begin{aligned}
& y_{-1}(t)=0, y_{n}(t)+\rho y_{n-1}(t)=0, t \geq 0 \\
& y(0)=w_{n}, \frac{d y_{n}(t)}{d t}=\kappa_{n}, n \in\left\{0,1_{n \ldots,} N-1\right\} .
\end{aligned}
$$

$\left\{y_{n}(t)\right\}_{n=1}^{N}$ find the solution and $k_{n}, h_{n}, a_{n}, b_{n}$ real parametres, $w_{n}, \kappa_{n} n=(0,1, \ldots, N-1)$ complex, equation (2) is the real number of the limit condition $\rho$, meets the following conditions. 
If a solution to the problem (1), (3) $\left\{y_{n}(t)\right\}_{n=1}^{N}$, (2) boundary conditions taking into account, they are obtained,

$$
\begin{gathered}
k_{0} \frac{d^{2} y_{n}(t)}{d t^{2}}=h_{0} \frac{d y_{0}(t)}{d t}+b_{0} y_{0}(t)+q a_{0} y_{1}(t), \\
k_{n} \frac{d^{2} y_{n}(t)}{d t^{2}}=h_{n} \frac{d y_{n}(t)}{d t}+a_{n-1} y_{n-1}(t)+b_{n} y_{n}(t)+q a_{n} y_{n+1}(t), \\
k_{N-1} \frac{d^{2} y_{N-1}(t)}{d t^{2}}=h_{N-1} \frac{d y_{N-1}(t)}{d t}+a_{N-2} y_{N-2}(t)+\left(b_{N-1}-h q a_{N-1}\right) y_{N-1}(t)
\end{gathered}
$$

This system, together with the initial conditions (3),

$$
\begin{aligned}
& y(t)=\left[\begin{array}{c}
y_{0}(t) \\
y_{1}(t) \\
\vdots \\
y_{N-1}(t)
\end{array}\right], w=\left[\begin{array}{c}
w_{0} \\
w_{1} \\
\vdots \\
w_{N-1}
\end{array}\right], \quad \kappa=\left[\begin{array}{c}
\kappa_{0} \\
\kappa_{1} \\
\vdots \\
\kappa_{N-1}
\end{array}\right], \\
& \mathrm{K}=\left[\begin{array}{ccc}
k_{0} & \cdots & 0 \\
\vdots & \ddots & \vdots \\
0 & \cdots & k_{N-1}
\end{array}\right], \mathrm{H}=\left[\begin{array}{ccc}
h_{0} & \cdots & 0 \\
\vdots & \ddots & \vdots \\
0 & \cdots & h_{N-1}
\end{array}\right] \\
& \mathrm{M}=\left[\begin{array}{ccccccccc}
b_{0} & q a_{0} & 0 & 0 & \cdot & . & \cdot & 0 & 0 \\
a_{0} & b_{1} & q a_{1} & 0 & \cdot & \cdot & \cdot & 0 & 0 \\
0 & a_{1} & b_{2} & q a_{2} & : & : & : & 0 & 0 \\
\cdot & \cdot & \cdot & : & : & : & \cdot & \cdot \\
0 & 0 & 0 & 0 & 0 & 0 & 0 & a_{N-2} & b_{N-1}-\rho a_{N-1}
\end{array}\right], \\
& \mathrm{K} \frac{d^{2} u(t)}{d t^{2}}=H \frac{d y_{n}(t)}{d t}+M u(t), \quad 0 \leq t<\infty \\
& y(0)=w, \frac{d y(0)}{d t}=\kappa
\end{aligned}
$$

we can write. Thus, the initial boundary value problem (1) - (3), the initial value problem (8), (9) the problem becomes equivalent [9]. Let

$$
y(t)=e^{a t} u
$$

be a non-trivial solution of the equation, where $\alpha$ is the complex constant, $u_{-}=\left\{u_{n}\right\}_{n=0}^{N-1} \in \mathrm{C}^{\mathbb{N}}$. Equation (10), (8) as well, they are obtained

$$
\left(\lambda^{2} \mathrm{~K}-\lambda \mathrm{H}-\mathrm{M}\right) u=0
$$

Let us record (11) the equation

$$
\begin{aligned}
& \left(\alpha^{2} \mathrm{k}_{\mathrm{n}}-\alpha \mathrm{h}_{\mathrm{n}}-\mathrm{b}_{\mathrm{n}}\right) \mathrm{u}_{\mathrm{n}}-a_{n-1} u_{n-1}-q a_{n} u_{n+1}=0, n=0,1, \ldots N-1 \\
& u_{-1}=0, u_{N}+h u_{N-1}=0
\end{aligned}
$$

it is equivalent to the boundary value problem. If the solution of the problem (12), (13) is $\left\{u_{n}\right\}_{n=-1}^{N}$, the $u=\left\{u_{n}\right\}_{n=0}^{N-1}$ vector provides the equation (11).

\section{Asymptotics of regular solutions of q-difference equations}

For $1>q>0$, we define the $q$-derivate of a real valued function $\mathrm{u}$ as

$$
D_{q} y(t)=\frac{y(t)-y(q))}{\llbracket(1-q) t}, D_{q} y(0)=\lim _{t \rightarrow 0} D_{q} y(t) .
$$

The higher order $q$ - derivatives are given by 


$$
D_{q}^{0} y(t)=y(t), D_{q}^{n} y(t)=D_{q} D_{q}^{n-1} y(t), \mathrm{n} \in \mathrm{N} .
$$

we will describe asymptotics of solutions of $q$-difference equations. A $q$-difference equation for a sequence $(y(1), y(2), y(3), \ldots)$ of smooth functions of $q$ has the form:

$$
\sum_{j=0}^{d} b_{j}\left(q^{k}, q\right) y(k+j, q)=0
$$

where $b j(v, u)$ are smooth functions and $y(k, q)=y(k)(q)$.

In the usual analytic theory of q-difference equations, $\mathrm{q}$ is a complex number inside or outside the unit disk. With this in mind, Q-difference equations are obtained from $q$-difference equations by the substitution $q=e^{2 \pi i a / n}$ where $\mathrm{Q}$ is a small nonnegative real number, that plays the role of Planck's constant.[10]. The characteristic polynomial of the $q$-difference equation (16) is

$$
P(v, \lambda)=\sum_{j=0}^{d} b j(v, 1) \lambda^{j}
$$

We will say that equation (16) is regular if

$$
\operatorname{Dsc}_{\lambda} P(v, \lambda) b 0(v, 1) b d(v, 1) \neq 0
$$

for all $\mathrm{v} \in \mathrm{S}^{1}$, where $D \operatorname{sc}_{\lambda} P(v, \lambda)$ is the discriminant of $P(v, \lambda)$, which is a polynomial in the coefficients of $P(v, \lambda)$. Let $\lambda_{1}(v), \ldots, \lambda d(v)$ denote the roots of the characteristic polynomial, which we call the eigenvalues of eq. (16). It turns out that eq.(16) is regular iff the eigenvalues $\lambda_{1}(v) \ldots, \lambda_{d}$ (v) never collide and never vanish, for every $\mathrm{v} \in \mathrm{S}^{1}$. Moreover, it follows by the implicit function theorem that the roots are smooth functions of $\mathrm{v}$ $\in \mathrm{S}^{1}$. Let $\mathrm{S}^{1}=\mathrm{U}_{\text {pep }} I p$ denote a partition of $\mathrm{S}^{1}$ into a finite union of closed arcs (with nonoverlapping interiors), such that the magnitude of the eigenvalues does not change in each arc. In other words, for each $p \in P$, there is a permutation $\sigma_{\mathrm{p}}$ of the set $\{1, \ldots, \mathrm{d}\}$ such that

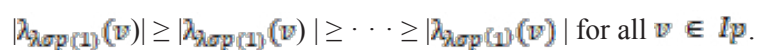

The following definition introduces a locally fundamental set of solutions of $q$-difference equations.

Fix a partition of $I$ as above. A set $\left\{\psi_{1}, \ldots, \psi_{\mathrm{d}}\right\}$ is a locally fundamental set of solutions of eq.(16) iff for every solution $\psi$ for every $p \in P$ and for every $m=1, \ldots, d$ there exist smooth functions $\mathrm{c} p \mathrm{~m}$ such that

$$
\psi(k, q)=c_{1}^{p}(q) \psi \sigma_{p}(1)\left(k_{s} q\right)+\cdots+c_{d}^{p}(q) \psi \sigma_{p}(d)(k, q) \text { for all }(\mathrm{k}, q): q^{\mathrm{k}} \in \mathrm{I}_{\mathrm{p}}
$$

Theorem 1.([11]) Assume that eq.(16) is regular. Then, there exists a locally fundamental set of solutions $\left\{\psi_{1}\right.$, . $\left.\ldots, \psi_{\mathrm{d}}\right\}$ such that

- For every $m=1, \ldots, d$ and $(k, q)$ such that $q^{\mathrm{k}} \in \mathrm{I}_{\mathrm{p}}$ we have

$$
\psi_{\mathrm{m}}\left(\mathrm{k}, e^{2 \pi i \alpha / n}\right)=\exp \left(\frac{n}{\alpha} \Phi_{\mathrm{m}}\left(\frac{k \Sigma}{n}, \frac{\sigma}{n}\right)\right) \text {. }
$$

- For some smooth functions $\Phi_{\mathrm{m}}$ with uniform (with respect to $x \in I=[0,1]$ ) asymptotic expansion

$$
\Phi \mathrm{m}\left(x_{s} \epsilon\right) \sim \sum_{s=0}^{\mathrm{s} s} \Phi \mathrm{m}_{s} \mathrm{~s}(x) \epsilon^{s}
$$

where $\varphi_{\mathrm{m}, \mathrm{s}} \in \mathrm{C}^{\infty}(\mathrm{I})$ for all $\mathrm{s}$

- and leading term

$$
\varphi_{\mathrm{m}, 0}(\mathrm{x})=\int_{0}^{x} \log \left(\lambda_{\mathrm{m}}\left(\mathrm{e}^{2 \pi \mathrm{it}}\right)\right) \mathrm{dt}
$$

where we have chosen a branch for the logarithm of $\lambda_{\mathrm{m}}$.

Definition 1. ([12]) We say that a formal series $\tilde{\psi}$ is a solution to (8) iff

$$
\frac{1}{\Psi(x, \epsilon)} \sum_{j=0}^{d} a_{j}(\mathrm{x}, \epsilon), \tilde{\psi}\left(x+j \epsilon_{,}, \epsilon\right)=0 \varepsilon \mathrm{C}^{\infty}(\mathrm{I})[[\mathrm{\epsilon}]]
$$

It is easy to see that if $\tilde{\psi}$ is a formal solution to (8), then the leading term $\varphi_{0}$ satisfies the equation

$$
\exp \left(\varphi^{\prime} 0(x)\right)=\lambda(x)
$$

where $\lambda(x)$ is an eigenvalue of (8).

The hypergeometrical basic series:

$$
2^{\Phi_{1}\left(a_{i}, b_{i} c_{i}, q_{i}, t\right)}=\sum_{n \geq 0} \frac{\left(\left[a_{i} q^{-1}\right)_{n}\left(b_{i} \cdot q^{-1}\right)_{n}\right.}{\left(c_{i} q^{-1}\right)_{n}\left(q^{-1} ; q^{-1}\right)_{n}} t^{n}
$$

is a convergent series, and it is the solution of the $q$-difference equation rational linear order two, 


$$
\sigma_{q}^{2} y-\lambda \sigma_{q} y+\mu y=0 \text { with }\left\{\begin{array}{c}
\lambda=\frac{(a+b) t-\left(1+\frac{5}{q}\right)}{a b t-\frac{5}{q}} \\
\mu=\frac{t-1}{a b t-\frac{E}{q}}
\end{array} .\right.
$$

It's a $q$-analogue (and a deformation) of Euler Gauss's classical hypergeometric symmetry,

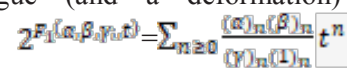

We find this last one by continuous limit (or "confluence") of coefficients. when $q \rightarrow 1$, with the constraints $a=q a, b=q \beta, c=q \gamma$. We'll see further in which precise direction one can confuse the functional equation (1) it self to a differential equation

$$
\sigma_{q}^{2} y-\tilde{\lambda} \sigma_{q} y+\tilde{\mu} y=0 \text { with }\left\{\begin{array}{c}
\tilde{\lambda}=\frac{(\alpha+\beta) t+(1-\gamma)}{1-t} \\
\tilde{\mu}=\frac{\alpha \beta t}{1-t}
\end{array} .\right.
$$

it admits for singularities 0,1 and $\infty$ only and that these singularities are regular. The Frobenius Method provides fundamental systems of solutions in 0 and in $\infty$ :

$$
\begin{aligned}
& \left\{\begin{array}{c}
2^{F_{1}\left(\alpha_{\beta} \beta_{2}, t\right)} \\
t^{1-Y} 2^{F_{1}(\alpha-\gamma+1, \beta-\gamma+1,2-\gamma, t)}
\end{array}\right. \\
& \left(\begin{array}{c}
\left.(1 / t)^{\alpha} 2^{F_{1}\left(\alpha_{j} \alpha-\gamma+1, \alpha-\beta+1\right.} \frac{1}{\mathrm{t}}\right) \\
\left.(1 / t)^{\beta} 2^{F_{1}(\beta, \beta-\gamma+1, \beta-\alpha+1} \frac{1}{\mathrm{t}}\right)
\end{array}\right)
\end{aligned}
$$

The analytical extension of a fundamental system solutions in the neighborhood of a singularity still provides a fundamental system solutions and therefore expresses itself from another fundamental system by an array of constants.

To determine the behavior of the solutions we give the following lemmas.

Lemma 1 . Define the function $f:(0, \infty) \rightarrow R$ by

$$
f(t)=\Phi\left(\frac{t}{q}-\frac{1}{q}\right)-\Phi\left(1-\frac{1}{t}\right) .
$$

Then $t \rightarrow f(t)$ is strictly increasing for $t>q^{1-1 / \alpha}$ and strictly decreasing for $0<t<q^{1-1 / \alpha}$.

Proof. The statement follows from the equality

$$
f^{\prime}(t)=\frac{a-1}{(t-1)^{2}}\left(q\left|\frac{t-1}{q}\right|^{\alpha}-\left|\frac{t-1}{t}\right|^{\alpha}\right) .
$$

Lemma 2. For $u \neq 0$ define the operator $P$ by

$$
P[u](t)=\Phi\left(\frac{u\left(q^{2} t\right)}{q u(q t)}-\frac{1}{q}\right)-\Phi\left(1-\frac{u(t)}{u(q t)}\right) .
$$

Then

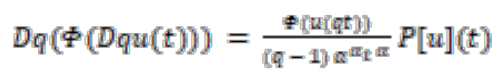

and equation (6) can be written as $P[u](t)=(q-1) \alpha^{\mathbb{E}_{L} \alpha} p(t)$ for $u \neq 0$.

Proof. From the $q$-derived formula

$$
D q(\Phi(D q u(t)))=D q\left(\Phi\left(\frac{u\langle q t)-u(t)}{(q-1) t}\right)\right)
$$




$$
\begin{aligned}
& =\frac{1}{(q-1) t}\left(\Phi\left(\left(\frac{u\left(q^{2} t\right)-u(q t)}{(q-1) q t}\right)-\Phi\left(\frac{u(q t)-u(t)}{(q-1) t}\right)\right)\right.
\end{aligned}
$$

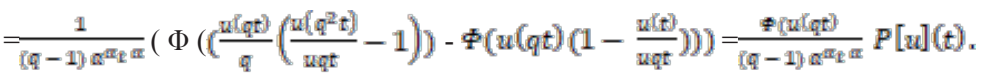

\section{Solving the Initial Boundary Value Problem}

In this section (4), (5) under the conditions of (1) - (3) the initial limit value the problem will be solved.

Theorem 2. (1) - (3) initial boundary value problem $C^{N}$ arbitrary $\left\{f_{n}\right\}_{n=0}^{N-1}$ and $g=\left\{g_{n}\right\}_{n=0}^{N-1}$ for $g$ g vectors

$$
u_{n}(t)=\sum_{j=1}^{2 N} \alpha_{j} e^{\lambda_{j} t} \varphi_{n}\left(\lambda_{j}\right) \quad(\mathrm{n}=-1,0,1, \ldots \mathrm{N})
$$

a unique $\left\{u_{n}(t)\right\}_{n=-1}^{N}$ has the solution. Where $\lambda_{1}, \lambda_{2, m}, \lambda_{2 N_{a}}$ the eigenvalues of the problem (11) and $\left\{\varphi_{n}(\lambda)\right\}_{n=-1}^{N}$ equation(12) the $q$-difference equation (13) is the solution that satisfies the initial conditions.

Proof. (20) formula gives a solution to the problem (1) - (3). Now that this solution is unique. We'll prove it. For this, the problems (1) - (9), which are equivalent to the problem (1) - (3) the problems

$$
\begin{aligned}
& \frac{d u(t)}{d t}=v(t) \\
& \frac{d v(t)}{d t}=C^{-1} R v(t)+C^{-1} J v(t), \\
& u(0)=f, v(0)=g
\end{aligned}
$$

and the last problem is

$$
\begin{aligned}
& \mathrm{A}=\left[\begin{array}{cc}
0 & 1 \\
C^{-1} J & C^{-1} R
\end{array}\right], \quad \mathrm{w}(\mathrm{t})=\left[\begin{array}{l}
w(t) \\
v(t)
\end{array}\right], w^{0}=\left[\begin{array}{l}
f \\
g
\end{array}\right], \text { to be } \\
& \frac{d w(t))}{d f t}=A w(t), 0 \leq t \leq \infty, \\
& w(0)=w^{0} .
\end{aligned}
$$

Thus, problem (8), (9); the problem (21), (22) becomes equivalent. That is, if $u(t)$ If the solution of the vector function (8), (9) is the solution, then

$$
\mathrm{w}(\mathrm{t})=\left[\begin{array}{l}
w(t) \\
w(t)
\end{array}\right]
$$

the vector function (21) is the solution to the problem (22) and, conversely,

$$
\mathrm{w}(\mathrm{t})=\left[\begin{array}{l}
u(t) \\
v(t)
\end{array}\right]
$$

if the vector function (21) is the solution of (22) then $v(t)=u^{f}(t)$ and $u(t)$ is the solution of the vector function (8), (9). So (21), (22) the problem is that for the first order linear differential equation system initial value problem and it is known that this problem is the only solution. So the theory is complete.

\section{Conclusion}

The examination of difference equations is indisputable in physics and mathematics. While solving the equations by discretizing the differential equations from one side, on the other hand, as mathematical models of many practical phenomena we also study the second - order self - adjoint linear homogeneous $q$ - difference equations. We examined the solutions under the initial conditions and gave them their general properties.

We also examined the global asymptotic behavior of solutions by applying the $q$-Caramate functions to the qdifference equations. These equations are a wave equation type with the second - order $q$ - difference derivative and therefore bear a physical meaning.

\section{References}

1. C. R. Adams, Annals of Mathematics, 2nd Ser. 30, 195 - 205 (1929)

2. F. V. Atkinson, Academic Press, New York (1964)

3. $\quad$ E. Bairamov ,Ö. Çakar, A.O. Çelebi, J. Math. Anal. Appl., 216, 303 - 320. (1997)

4. $\quad$ E. Bairamov, Ö. Çakar, A.M. Krall, J. Diff. Equations, 151, 268 - 289. (1999)

5. F.H. Jackson, Amer. J. Math. 32, 305 - 314.(1910) 


\section{S. Elaydi, Springer - Verlag, New York (1996)}

7. G.S. Guseinov, G. Oturanç, Turkish J. Math. 21, 409 - 422 (1997)

8. $\quad$ G.S. Guseinov, M.Terziler, Linear Algebra and Its Applications, 259, 329 - 346.(1997)

9. A. Hüseynov, Ankara University Inst. of Science and Tec.(2006)

10. R. Kashaev, Modern Phys. Lett. A 39, 269-275. (1997).

11. O. Costin, R. Costin, SIAM J. Math. Anal. 27, 110-134. (1996)

12. S. Garoufalidis, J.S. Geronimo, arXiv:math/0405331 [math.QA], (2006) 